\title{
Estresse percebido e nível de atividade física em docentes de um Instituto Federal
}

\author{
Stress and level of physical activity perceived in \\ teachers of a Federal Institute
}

Thaizi CAMpos Barbosa ${ }^{\mathrm{I}}$ ANDRÉ PONTES SILVA ${ }^{I I}$ Fernando Rodrigues Peixoto QUARESMA ${ }^{\text {III }}$

ERIKa da Silva Maciel ${ }^{\text {III }}$

${ }^{1}$ Instituto de Ensino e Pesquisa Objetivo (IEPO). Palmas/TO II Universidade Federal do Maranhão (UFMA), São Luís/MA - Brasil III Universidade Federal do Tocantins (UFT). Palmas/TO -Brasil.
Resumo Introdução: a atividade laboral pode ser extremamente desgastante para o profissional, pelo excesso de cobrança, carga horária elevada e sobrecarga de trabalho. A rotina dos docentes também não difere disso, sendo que a profissão é considerada uma das mais estressantes, pois causa efeitos evidentes na saúde e no desempenho profissional destes. Porém, atitudes simples, como a prática de Atividade Física, podem contribuir para a melhora dessa condição.

Objetivo: avaliar o Nível de Atividade Física e Estresse Percebido, bem como, sua correlação, em docentes de um Instituto Federal da região norte do Brasil.

Métodos: um estudo transversal de caráter quantitativo e com questionários em formato on-line. As variáveis de exposição foram características sociodemográficas, e as variáveis de desfecho a atividade física e estresse percebido. O projeto teve aprovação no Comitê de Ética.

Resultados: participaram do estudo 68 docentes, sendo sua maioria Mestres. Quanto ao Nível de Atividade Física, a maior parte foi classificada como ativos e muito ativos. Docentes mestres expressam média de estresse superior quando comparados aos demais, porém os doutores apresentam maior escore de Equivalente Metabólico, se mostrando mais ativos. Houve correlação inversa entre as variáveis de Equivalente Metabólico e Estresse Percebido, evidenciando que quanto maior a prática de atividades físicas, tanto menor o estresse. Conclusão: foi observado que docentes Mestres relacionam-se com a maior média de estresse percebido; docentes doutores correspondem à maior média de Equivalente Metabólico; houve correlação inversa estatisticamente significativa entre as variáveis.

Palavras-chave: Exercício Físico. Equivalente Metabólico. Estresse Ocupacional. Saúde do Trabalhador. Docentes.

ABSTRACT Introduction: the work activity can be extremely exhausting for the professional, due to overcharging, high hours and overload of work. The teacher's routine also does not differ from this, as the profession is considered one of the most stressful, as it causes evident effects on their health and professional performance. However, simple attitudes, such as the practice of Physical Activity can contribute to the improvement of this condition.

Objective: to evaluate the Level of Physical Activity and Perceived Stress, as well as it's correlation, in teacher's of a Federal Institute in the northern region of Brazil. 


\begin{abstract}
Methods: a cross-sectional study of a quantitative character and with questionnaires in online format. The exposure variables were socio-demographic characteristics and the outcome variables, physical activity and perceived stress. The project was approved by the Ethics Committee. Results: 68 teachers participated in the study, most of whom were Masters. As for the Physical Activity Level, most were classified as active and very active. Master teacher's express a higher average stress when compared to the others, but doctors have a higher Metabolic Equivalent score, proving to be more active. There was an inverse correlation between the variables of Metabolic Equivalent and Perceived Stress, showing that the greater the practice of physical activities, the lower the stress.

Conclusion: it was observed that: Master teacher's are related to the highest average of perceived stress; doctoral professors correspond to the highest average of Metabolic Equivalent; there was a statistically significant inverse correlation between variables.
\end{abstract}

Keywords: Exercise. Metabolic Equivalent. Occupational Stress. Occupational Health Faculty.

\section{INTRODUÇÃO}

A rotina laboral, isto é, a ocupação de uma pessoa, pode ser intensa e estressante em muitos casos, pois alguns empregadores cobram muito de seus funcionários, exigindo o máximo de rendimento, com sobrecarga de trabalho e jornada extenuante. ${ }^{1}$ Nos casos em que ocorrem adoecimentos e alterações do comportamento fisiológico do corpo e mente desses indivíduos sobrecarregados de trabalho, seu expediente de ocupação nem sempre é levado em consideração em uma consulta, diagnóstico ou tratamento. ${ }^{2}$ Todavia, essas condições de trabalho afetam negativamente na saúde física e mental do trabalhador, causando fadiga e estresse, uma resposta fisiológica aos estímulos estressores. ${ }^{3}$

Profissionais da saúde, na tentativa de obter uma explicação plausível para as condições de saúde de seus pacientes, ao se depararem com os problemas físicos e psíquicos apresentados - fadiga, estresse e afins -, concentram-se apenas na investigação da herança genética, condições patológicas explícitas, histórico familiar, situação afetiva e comorbidades dos pacientes. ${ }^{4}$ Em geral, estas são consideradas as principais referências que subsidiam as explicações obtidas nos diagnósticos de consultas, assim, o estresse oriundo do ambiente de trabalho do paciente - chamado de estresse ocupacional -, nem sempre faz parte da anamnese de investigação em condição de saúde humana. ${ }^{5}$

Porém, esse cenário de investigação da saúde do trabalhador tem sido discutido na literatura desde a segunda metade do século XX, e, a abordagem biopsicossocial tem dado maior atenção aos aspectos relacionados à dimensão do trabalho, relacionando-o ao estresse ocupacional e percepção de qualidade de vida. ${ }^{2}$

Há estudos que indicam o impacto negativo do estresse ocupacional sobre a saúde das pessoas, associando-o à resistência à insulina, dislipidemia, hipertensão arterial, doenças cardiovasculares e osteomusculares, distúrbios psíquicos, depressão, entre outros. ${ }^{6,8}$

Entre as profissões que mais geram estresse ocupacional, ensinar é uma delas, pois causa efeitos evidentes na saúde física, men- 
tal e no desempenho profissional dos professores, em virtude das inúmeras situações estressoras no contexto educacional. ${ }^{9,2}$

Nas últimas décadas do século $\mathrm{XX}$, e início do século XXI, foi mostrado o efeito do estresse ocupacional sobre a saúde em docentes, onde se destacaram os fatores psicológicos - como ansiedade, depressão, irritabilidade, hostilidade, exaustão emocional - e efeitos sistêmicos - como doenças cardiovasculares, labirintites, faringites, neuroses, fadiga, insônia e tensão nervosa. ${ }^{10}$

No entanto, o estresse pode ser reduzido com medidas simples, entre essas a prática regular de Atividade Física (AF), que contribui ainda com a minimização de outros agravos à saúde influenciados pelo estresse - por exemplo os efeitos psicológicos e sistêmicos supracitadas. ${ }^{11}$ Além disso, há uma correlação positiva entre bem-estar físico, emocional e social e a prática regular de $\mathrm{AF}^{12}$ Ademais, é possível observar mudanças nos componentes antropométricos, cognitivos e psicossociais dos praticantes regulares de $\mathrm{AF}^{13}$

No Brasil, há escassez de estudos sobre o estresse ocupacional. ${ }^{9}$ Considerando que o estresse tem impacto negativo na saúde dos professores, e que a $\mathrm{AF}$ regular pode ser uma alternativa capaz de promover melhor percepção de qualidade de vida aos docentes, é possível emitir a seguinte indagação: qual é o Nível de Atividade Física (NAF) e Estresse Percebido (EP) dos docentes de um Instituto Federal da região norte do Brasil?

Com base no que foi exposto, o objetivo da pesquisa foi avaliar o NAF e EP, bem como, sua relação, dos docentes de um Instituto Federal da região norte do Brasil.

\section{Métodos}

Um estudo transversal de caráter quantitativo. O projeto de pesquisa foi subme- tido e aprovada pelo Comitê de Ética em Pesquisas com Seres Humanos (CAAE) 55072016.0.0000.5516. A coleta aconteceu entre agosto e outubro de 2016.

O questionário foi preenchido - em formato on-line utilizando a plataforma Google Forms -, por docentes de um Instituto Federal na região Norte do país. Variáveis de exposição: características sociodemográficas. Variáveis de desfecho: NAF e EP.

$\mathrm{O}$ artigo seguiu as recomendações da Strengthening the Reporting of Observational Studies in Epidemiology (STROBE), ${ }^{14,15}$ que objetiva melhorar a qualidade da escrita e facilitar a leitura crítica por parte dos editores, revisores e leitores.

Para participar da pesquisa, convidamos todos os docentes (216) que compõem o quadro da instituição em questão. A proposta, com o Termo de Consentimento Livre Esclarecido (TCLE), foi enviada para o e-mail institucional de cada professor. Somente após concordância notificada, em resposta ao convite, enviamos o link com o formulário on-line para preenchimento dos instrumentos da pesquisa. Aqueles profissionais que se encontravam de férias, licença e afins, recusaram assinar o TCLE (141 docentes) e outliers por falta de informações contidas nos questionários ( 7 docentes), foram excluídos da pesquisa.

O Metabolic Equivalent of Task (MET) e EP - de desfecho do estudo -, estão apresentados como variáveis quantitativas. Já a variável de NAF foi exposta, segundo sua classificação: muito ativo, ativo, insuficientemente ativo e sedentário.

As variáveis sociodemográficas - como sexo, nível de escolaridade, quantidade de filhos, naturalidade, etnia, renda mensal e idade -, são consideradas como controle. Para as condições sociodemográficas, uti- 
lizamos o instrumento desenvolvido pelos pesquisadores contendo questões pessoais - como, sexo, idade, nível de escolaridade, quantidade de filhos, entre outros.

Para avaliação do NAF, foi utilizado o International Physical Activity Questionnaire (IPAQ), versão 8, forma curta e semana normal. O instrumento é composto por oito questões que abordam a intensidade, frequência e duração de atividades físicas realizadas na semana anterior e divididas em caminhada, atividades moderadas, vigorosas e sedentárias, possibilitando classificar o indivíduo em: sedentário, insuficientemente ativo, ativo e muito ativo. ${ }^{16}$

O MET é obtido por meio do múltiplo da taxa metabólica basal, que se refere à energia suficiente para um indivíduo se manter em repouso, nesse sentido, o gasto de energia em MET significa predizer o número de vezes pelo qual o metabolismo de repouso foi multiplicado durante uma atividade. Por exemplo: pedalar a três METs implica em gasto calórico três vezes maior que o que vigora em repouso na taxa metabólica basal. ${ }^{17,18}$

$\mathrm{O}$ instrumento utilizado para fazer essa avaliação foi o mesmo do NAF, pois seu resultado é apresentado em valor quantitativo de MET, sendo que cada atividade tem seu valor de dispêndio de energia (caminhada é de 3,3 , as atividades moderadas 4,0 e as vigorosas 8,0 ). Esse valor é multiplicado pela frequência semanal e duração da atividade, que representa o gasto calórico em MET minuto/semana. ${ }^{19}$

O EP foi avaliado por meio do instrumento Escala de Percepção do Estresse - 10 (EPS-10) composto por 10 perguntas de múltiplas escolhas. Segundo a EPS-10, quanto maior a pontuação tanto maior a percepção de estresse $e^{20}$ validado e aplicado para a população brasileira. ${ }^{21}$

A variável quantitativa de EP está exposta com seus dados brutos, sem nenhuma categorização ou agrupamento, ou seja, não consta classificação. A variável de MET está descrita com seus dados brutos e em sua classificação de NAF como complemento. O EP e MET estão apresentados dessa maneira para estimar as relações diretas entre essas variáveis de desfecho.

Para análise de dados, consideramos as recomendações dos questionários e, posteriormente, submetemos a análise descritiva por meio do software Statistical Package for the Social Sciences (SPSS) versão 20.0. As variáveis qualitativas estão descritas por frequência e percentuais. As variáveis quantitativas, após aplicação do teste de normalidade Shapiro-Wilk, foram expressadas por média e desvio padrão, quando apresentarem aderência à distribuição normal $(\mathrm{p}>0,05)$ e medianas e percentis quando não mostrarem aderência à distribuição normal $(\mathrm{p}<0,05)$. $\mathrm{O}$ teste de correlação de Spearman $(\mathrm{p}<0,05)$ foi utilizado para analisar a relação entre estresse percebido e equivalente metabólico.

\section{Resultados}

Participaram da pesquisa 68 docentes que atuam na instituição de ensino, sendo $51,47 \%$ dos indivíduos do sexo feminino, casados $(73,53 \%)$, com dois filhos $(35,30)$ declarados brancos $(45,59 \%)$ naturais da Região Nordeste (27,93\%), com renda mensal acima de 10 salários mínimos $(44,12 \%)$ e com escolaridade de Mestre (57,35\%), Tabela 1. 
Tabela 1 - Perfil Sociodemográfico.

\begin{tabular}{|c|c|c|}
\hline Variáveis & $\mathbf{n}$ & $\%$ \\
\hline \multicolumn{3}{|l|}{ Sexo } \\
\hline Feminino & 35 & 51,47 \\
\hline Masculino & 33 & 48,43 \\
\hline \multicolumn{3}{|l|}{ Estado Civil } \\
\hline Solteiro & 13 & 19,12 \\
\hline Casado & 50 & 73,53 \\
\hline Separado & 4 & 5,88 \\
\hline Outros & 1 & 1,47 \\
\hline \multicolumn{3}{|l|}{ Filhos } \\
\hline Nenhum & 21 & 30,88 \\
\hline 1 filho & 17 & 25,00 \\
\hline 2 filhos & 24 & 35,30 \\
\hline 3 filhos & 6 & 8,82 \\
\hline \multicolumn{3}{|l|}{ Etnia } \\
\hline Preto & 5 & 8,34 \\
\hline Branco & 31 & 45,59 \\
\hline Pardo & 27 & 39,71 \\
\hline Mestiço & 1 & 1,47 \\
\hline Branco/pardo & 1 & 1,47 \\
\hline Amarelo & 3 & 4,42 \\
\hline \multicolumn{3}{|l|}{ Naturalidade } \\
\hline Nordeste & 19 & 27,93 \\
\hline Sudeste & 14 & 20,59 \\
\hline Centro-oeste & 16 & 23,53 \\
\hline Sul & 9 & 13,24 \\
\hline Norte & 9 & 13,24 \\
\hline Estrangeiro & 1 & 1,47 \\
\hline \multicolumn{3}{|l|}{ Renda mensal } \\
\hline 3 a 5 salários mínimos & 5 & 7,35 \\
\hline 5 a 8 salários mínimos & 16 & 23,53 \\
\hline 8 a 10 salários mínimos & 17 & 25,00 \\
\hline $\begin{array}{l}\text { Acima de } 10 \text { salários } \\
\text { mínimos }\end{array}$ & 30 & 44,12 \\
\hline \multicolumn{3}{|l|}{ Escolaridade } \\
\hline Mestre & 39 & 57,35 \\
\hline Doutor & 16 & 23,53 \\
\hline Especialista & 13 & 19,12 \\
\hline
\end{tabular}

A média de idade entre os avaliados foi de 40,81 $( \pm 7,16)$, em relação ao EP, a média geral foi de 19,88 ( $\pm 5,07)$ e o Equivalente Metabólico da Tarefa (MET) dos docentes com mediana de 1401,5 ( $\pm 0 ; 2739)$, Tabela 2 .

Tabela 2 - Análise descritiva das variáveis do estudo.

\begin{tabular}{l|c|c}
\hline Variáveis & Média & $\begin{array}{c}\text { Desvio Padrão } \\
( \pm)\end{array}$ \\
\hline Idade & 40,81 & $7,16)$ \\
\hline Estresse percebido & 19,88 & 5,07 \\
\hline & Mediana & $\begin{array}{c}\text { Percentis (p25; } \\
\text { p75) }\end{array}$ \\
\hline MET & 1401,5 & $0 ; 2739$ \\
\hline
\end{tabular}

Em relação ao NAF, foi observado que 66,18\% dos participantes não são sedentários, caracterizados nessa pesquisa como ativos $(44,12 \%)$ e muito ativos $(22,06 \%)$. Todavia, o número de participantes insuficientemente ativos $(10,29 \%)$ e sedentários $(23,53)$ é relativamente alto $(33,82 \%)$, Tabela 3 .

Tabela 3 - Classificação do nível de atividade física.

\begin{tabular}{l|c|c}
\hline Classificação & $\mathrm{n}$ & $\%$ \\
\hline Muito ativo & 15 & 22,06 \\
Ativo & 30 & 44,12 \\
\hline Insuficientemente ativo & 7 & 10,29 \\
\hline Sedentário & 16 & 23,53 \\
\hline
\end{tabular}

Quanto ao nível de escolaridade comparado com o estresse percebido e equivalente metabólico, pode-se observar que os mestres possuem maior média de EP, com 21,03 ( \pm $4,49)$ em relação aos demais níveis, tendo o MET mais baixo, com mediana de 1295 . Enquanto os doutores apresentam menor média de EP e maior mediana de MET (Tabela 4). 
Tabela 4 - Descrição do Estresse Percebido e Equivalente Metabólico por Escolaridade.

\begin{tabular}{lccccc}
\hline & \multicolumn{2}{c}{ Estresse Percebido } & \multicolumn{2}{c}{ Equivalente metabólico } \\
\hline Escolaridade & $\mathrm{n}$ & Média & Desvio Padrão $( \pm)$ & Mediana & Percentis \\
& & & & & $(\mathrm{p} 25 ; \mathrm{p} 75)$ \\
\hline Especialista & 13 & 18,46 & 4,46 & 1752 & $516 ; 2640$ \\
Mestre & 39 & 21,03 & 4,49 & 1295 & $99 ; 2652$ \\
\hline Doutor & 16 & 18,25 & 6,31 & 2133 & $457 ; 3432$ \\
\hline
\end{tabular}

O estudo apresentou que, nessa análise, houve uma correlação negativa e estatisticamente significativa entre as variáveis de EP e MET pelo teste de Spearman (rho=-0,2623; $\mathrm{p}=0,0307)$, ou seja, quanto maior o MET tanto menor o EP.

\section{Discussão}

O presente estudo envolveu uma avaliação do NAF e EP de 68 docentes de uma instituição de ensino médio-técnico, tecnólogo e superior. Apesar de exibir uma correlação fraca entre as variáveis estudadas, o resultado indica uma relação inversa em que, quanto maior o MET tanto menor será a percepção de estresse dos participantes.

Em relação ao $\mathrm{EP}$, constatou-se que os docentes têm uma média de percepção de estresse de 19,88 ( $\pm 5,07)$, esse escore pode ser comparado com a tabela normativa da população norte-americana utilizada por Cohen et al. ${ }^{20}$ Cohen, ${ }^{22}$ ou ainda, com a população de professores do Sul do Brasil utilizada por Petroski ${ }^{23}$ e Reis et al. ${ }^{24}$ em que quanto maior o valor descrito, tanto maior será o estresse (considerando o número 0 como referência de ausência de EP). Os achados dessa pesquisa apresentam um elevado nível de EP entre os docentes observados/entrevistados. Isso

pode ser explicado pelo fato de os professores terem, além da carga horária de trabalho normal, atividades administrativas e produções científicas.25 Já sobre EP em relação à escolaridade, os docentes que possuem mestrado exibiram a maior média quando comparados aos especialistas e doutores, resultado que difere de outro estudo com a mesma população em que os achados mostram doutores como detentores do maior estresse. ${ }^{25}$

Porém, quando esses dados são observados com os valores de MET, é possível verificar que os Mestres possuem o menor valor de MET, ou seja, o menor NAF, enquanto os doutores apresentam maior valor de MET entre as categorias. Esse resultado permite dizer que o dado anterior - em que os Mestres são mais estressados -, sugere que a prática de AF contribui para redução do estresse. ${ }^{11,13,26}$

Em relação à NAF dos participantes, a maioria foi considerada ativa $(44,12 \%)$, porém, o número de participantes classificados como insuficientemente ativos (10,29\%) e sedentários $(23,53 \%)$ foi elevado, apresentando assim uma preocupação com esse cenário. Estudos com docentes demonstram que esses profissionais tendem à NAF irregulares e inadequados, ${ }^{26,25,27,28,29}$ sendo a falta de tempo a justificativa mais utilizada para a pouca prática $\mathrm{AF}^{30}$ 
O contexto da prática de AF insuficiente contribui para o quadro de estresse dos docentes, pois é sabido que a prática de $\mathrm{AF}$ tem impacto positivo na saúde e na manutenção e recuperação de condições patológicas de uma população, relacionando-se à diminuição do EP, aumento da qualidade de vida, prevenção e tratamento de doenças degenerativas não transmissíveis, como as cardiovasculares citadas na introdução, por exemplo. ${ }^{31,11,12,13}$ Conforme observado no presente estudo, há uma correlação inversa entre AF e o EP, assim como em outros estudos, ${ }^{25,29}$ certificando que a prática de AF se relaciona com saúde humana de forma generalizada. Porém, mesmo encontrando subsídios na literatura para sustentar a importância da atividade física para o bem-estar físico, mental e social, o modelo de educação no Brasil parece expor o docente a situações de estresse ocupacional e baixo NAF provenientes do desempenho das funções profissionais, pois as atividades acadêmicas/escolares vão além da carga horária expressada na legislação, não havendo tempo para cuidar de si e, adotar hábitos de vida saudáveis, por exemplo, a prática regular de $\mathrm{AF}^{32}$

Porém, mesmo encontrando subsídios na literatura para sustentar a importância da atividade física para o bem-estar físico, mental e social, o modelo de educação no Brasil parece expor o docente a situações de estresse ocupacional e baixo NAF provenientes do desempenho das funções profis- sionais, pois as atividades acadêmicas/escolares vão além da carga horária expressada na legislação, não havendo tempo para cuidar de si e, adotar hábitos de vida saudáveis, por exemplo, a prática regular de $\mathrm{AF}^{32}$

\section{CONSIDERAÇões FINAIS}

Este estudo identificou que docentes com titulação de mestrado apresentaram maior média de estresse percebido, quando comparados aos demais. Houve correlação inversa estatisticamente significativa entre as variáveis MET e a EP dos participantes, indicando que pessoas fisicamente ativas podem exibir menos estresse. Sabendo da importância desses resultados, sugere-se estudos sobre efeitos de programas de exercícios físicos nessa população.

A respeito das limitações deste trabalho, vale destacar que se trata de um estudo transversal, portanto, não é possível identificar a causa do estresse nos participantes. Além disso, os dados podem exibir vieses comuns à amostra, pois talvez não representem bem toda a população investigada (em virtude do baixo tamanho do $n$ ), todavia, para minimizar os vieses contatamos e convidamos todos os professores da instituição. Apesar do estudo ter se realizado em uma única instituição, esses dados demonstram a importância da pesquisa sobre as variáveis de EP e AF em docentes, e, nesse sentido, podem ser aplicados em outras localizações. 


\section{REFERÊNCIAS}

1. Silva NC da, Ferreira JVB, Albuquerque TC, Rodrigues MR, Medeiros MF. Transtornos à saúde mental relacionados à intensa rotina de trabalho do enfermeiro: uma revisão bibliográfica. Rev Eletrônica Estácio Saúde [Internet]. 2016; 5 (2): 107-22.

2. Borsoi F., Cristina I. Da Relação Entre Trabalho E Saúdeà Relação Entre Trabalho E Saúde Mental. Psicol Soc [Internet]. 2007; 19 (1).

3. Bessa de Andrade Fernandes SM, Medeiros SM de, Ribeiro LM. Estresse ocupacional e o mundo do trabalho atual: repercussões na vida cotidiana das enfermeiras. Rev Eletrônica Enferm [Internet]. 2009; 10 (2): 414-27.

4. Lipp MEN. O Estresse do Professor. Campinas: Papirus; 2003, 136 p.

5. Codo W. O trabalho enlouquece? um encontro entre a clínica e o trabalho. Rio de Janeiro: Vozes; 2004, 238 p.

6. Flynn N., James JE. Relative effects of demand and control on task-related cardiovascular reactivity, task perceptions, performance accuracy, and mood. Int J Psychophysiol [Internet]. 2008; 72 (2): 217-27.

7. Schnall PL, Landsbergis PA, Baker D. Job Strain and Cardiovascular Disease. Annu Rev Publi<' Heal 1994 [Internet]. 1994; 15 (9): 381-411.

8. Van Der Wal RAB, Wallage J., Bucx MJL. Occupational stress, burnout and personality in anesthesiologists. Curr Opin Anaesthesiol [Internet]. 2018, jun.; 31 (3): 351-6.

9. Zonatto VC da S., Weber A, Nascimento JC. Efeitos da Participação Orçamentária na Assimetria Informacional, Estresse Ocupacional e Desempenho Gerencial. Rev Adm Contemp [Internet]. 2019, fev.; 23 (1): 67-91.

10. Cooper G. Handbook of stress, medicine and health. Nova York: CRC; 1996.

11. Bertoldi AD, Hallal PC, Barros AJ. Physical activity and medicine use: Evidence from a population-based study. BMC Public Health [Internet]. 2006, Dec. 6; 6 (1): 224.

12. Tecnol U., Federal G., Paran DO, Educa MDE, Curso S., Em DEB, et al. Associação entre atividade física, qualidade de vida e estresse de docentes do ensino superior do departamento acadêmico de educação física da UTFPR campus Curitiba. (Bachelor's thesis, Univ Tecnológica Fed do Paraná) [Internet]. 2017.

13. Nelson ME, Rejeski WJ, Blair SN, Duncan PW, Judge JO, King AC. et al. Physical Activity and Public Health in Older Adults. Med Sci Sport Exerc [Internet]. 2007, Aug.; 39 (8): 1.435-45.

14. Malta M., Cardoso LO, Bastos FI, Magnanini MMF, Silva CMFP da. Iniciativa STROBE: subsídios para a comunicação de estudos observacionais. Rev Saude Publica. 2010; 44 (3): 559-65.

15. Von Elm E., Altman DG, Egger M., Pocock SJ, Gøtzsche PC, Vandenbroucke JP, et al. The Strengthening the Reporting of Observational Studies in Epidemiology (STROBE) Statement: Guidelines for Reporting Observational Studies. PLoS Med. 2007, Oct.; 4 (10): e 296.

16. Matsudo S., Araújo T., Matsudo V., Andrade D., Andrade E., Oliveira L. et al., Questionário internacional de ativi da.de fisica (IPAQ): estudo de vall dade e reprodutibilidade no Brasil. Atividade Física \& Saúde. 2001; 6 (2): 5-18.

17. Ravagnani C. de FC, Melo FCL, Ravagnani FCP, Burini FHP, Burini RC. Estimativa do equivalente metabólico (MET) de um protocolo de exercícios físicos baseada na calorimetria indireta. Rev Bras Med do Esporte [Internet]. 2013, Apr.; 19 (2): 134-8.

18. Crisp AH, Verlengia R., Oliveira MRM. Limitações da utilização do equivalente metabólico (MET) para estimativa do gasto energético em atividades físicas. Rev Bras Ciência e Mov [Internet]. 2014; 22 (3): 148-53. 
19. Almeida LAB de, Pitanga FJG, Freitas MM, Pitanga CPS, Dantas EHMD, Beck CC. Gasto Calórico Dos Diferentes Domínios De Atividade Física Como Preditor Da. 2012; (Dm): 17-21.

20. Cohen S., Kamarck T., Mermelstein R. A Global Measure of Perceived Stress A Global Measure of Perceived Stress. 1983; 24 (4): 385-96.

21. Reis RS, Hino AAF, Añez CRR. Perceived Stress Scale: Reliability and Validity Study in Brazil. J Health Psychol. 2010; 15 (1): 107-14.

22. Cohen S. Perceived Stress Scale (PSS). Encycl Behav Med. 1994; 1.454-5.

23. Petroski EC. Qualidade de vida no trabalho e suas relações com estresse, nível de atividade física e risco coronariano de professores universitários. Univ Fed St Catarina - Tese, 2005; $1-163$.

24. Reis EJFB dos, Carvalho FM, Araújo TM de, Porto LA, Silvany Neto AM. Trabalho e distúrbios psíquicos em professores da rede municipal de Vitória da Conquista, Bahia, Brasil. Cad Saude Publica [Internet]. 2005, Oct.; 21 (5): 1.480-90.

25. Soares MB, Mafra SCT, De Faria ER. Factors associated with perceived stress among professors at a federal public university. Rev Bras Med do Trab [Internet]. 2019; 17 (1): 90-8.

26. Santos MPG dos, Silva KKD da. Níveis de estresse e qualidade de vida de professores do ensino superior. Rev Enferm da UFSM. 2017; 7 (4): 656.

27. Reis ASF, De Oliveira BG, Bomfim EDS, Boery RNS de O., Boery EN. Avaliação Da Influência Do Nível De Atividade Física Na Qualidade De Vida Do Professor Universitário. Arq Ciências da Saúde. 2017; 24 (1): 75.

28. Dias J., Dusmann Junior M., Costa MAR, Francisqueti V., Higarashi IH. Physical activities practicing among scholar professors: focus on their quality of life. Esc Anna Nery, 2017; 21 (4): $1-6$.

29. Barruffini ACC, Silva VO, Sousa IF de, Bastos GCFC, Silva AMTC, Almeida RJ de. Fatores associados aos níveis de estresse laboral em professores de um curso de medicina. Saúde Coletiva (Barueri) [Internet]. 2019; (51): 2.013-9.

30. Rhodes RE, Smith NEI. Personality correlates of physical activity: a review and meta-analysis. $\mathbf{B r}$ J Sports Med [Internet]. 2006. Dec. 1; 40 (12): 958-65.

31. Carlos MBB. Estudo comparativo entre a percepção da qualidade de vida e o nível de estresse em indivíduos praticantes de pilates solo, hidroginástica e sedentários. TCC Grad Formiga/MG Cent Univ Formiga, [Internet]. 2013; 0-57.

32. Freitas EDS, Senra LX. Breve análise bibliométrica sobre a síndrome de Bornout em professores universitários. 2014.

Agradecimentos

Manifestamos agradecimento aos docentes do IFTO pela confiança, apoio e participação neste projeto.

\section{DAdos dos AUTORES}

\section{Thaizi Campos Barbosa}

Docente do curso de Educação Física do Instituto de Ensino e Pesquisa - Objetivo (IEPO). Palmas/ TO. thaizi@gmail.com

\section{André Pontes Silva}

Mestrando do Programa de Pós-Graduação em Saúde do Adulto da Universidade Federal do Maranhão (UFMA). São Luís/MA - Brasil. contato.andrepsilva@gmail.com 


\section{Fernando Rodrigues Peixoto Quaresma}

Docente do Curso de Enfermagem da Universidade Federal do Tocantins (UFT). Palmas/TO -Brasil. ferodriguesto@gmail.com

\section{ERika da Silva Maciel}

Docente do Curso de Educação Física da Universidade Federal do Tocantins (UFT). Miracema/TO

- Brasil.erikasmaciel@gmail.com

Submetido em: 15-4-2020

Aceito em: 15-8-2020 